\title{
PERIODIC SOLUTIONS OF A CLASS \\ OF NON-AUTONOMOUS SECOND-ORDER \\ DIFFERENTIAL INCLUSIONS SYSTEMS
}

DANIEL PAŞCA

Received 15 March 2001

Using an abstract framework due to Clarke (1999), we prove the existence of periodic solutions for second-order differential inclusions systems.

\section{Introduction}

Consider the second-order system

$$
\begin{gathered}
\ddot{u}(t)=\nabla F(t, u(t)) \quad \text { a.e. } t \in[0, T], \\
u(0)-u(T)=\dot{u}(0)-\dot{u}(T)=0,
\end{gathered}
$$

where $T>0$ and $F:[0, T] \times \mathbb{R}^{n} \rightarrow \mathbb{R}$ satisfies the following assumption:

(A) $F(t, x)$ is measurable in $t$ for each $x \in \mathbb{R}^{n}$ and continuously differentiable in $x$ for a.e. $t \in[0, T]$, and there exist $a \in C\left(\mathbb{R}^{+}, \mathbb{R}^{+}\right), b \in L^{1}\left(0, T ; \mathbb{R}^{+}\right)$ such that

$$
\begin{gathered}
|F(t, x)| \leq a(\|x\|) b(t), \\
\|\nabla F(t, x)\| \leq a(\|x\|) b(t),
\end{gathered}
$$

for all $x \in \mathbb{R}^{n}$ and a.e. $t \in[0, T]$.

Wu and Tang in [4] proved the existence of solutions for problem (1.1) when $F=F_{1}+F_{2}$ and $F_{1}, F_{2}$ satisfy some assumptions. Now we will consider problem (1.1) in a more general sense. More precisely, our results represent the extensions to systems with discontinuity (we consider the generalized gradients unlike continuously gradient in classical results). 


\section{Main results}

Consider the second-order differential inclusions systems

$$
\begin{gathered}
\ddot{u}(t) \in \partial F(t, u(t)) \quad \text { a.e. } t \in[0, T], \\
u(0)-u(T)=\dot{u}(0)-\dot{u}(T)=0,
\end{gathered}
$$

where $T>0, F:[0, T] \times \mathbb{R}^{n} \rightarrow \mathbb{R}$ and $\partial$ denotes the Clarke subdifferential.

We suppose that $F=F_{1}+F_{2}$ and $F_{1}, F_{2}$ satisfy the following assumption:

(A') $F_{1}, F_{2}$ are measurable in $t$ for each $x \in \mathbb{R}^{n}$, at least $F_{1}$ or $F_{2}$ are strictly differentiable in $x$ and there exist $k_{1} \in L^{2}(0, T ; \mathbb{R})$ and $k_{2} \in L^{2}(0, T ; \mathbb{R})$ such that

$$
\begin{aligned}
& \left|F_{1}\left(t, x_{1}\right)-F_{1}\left(t, x_{2}\right)\right| \leq k_{1}(t)\left\|x_{1}-x_{2}\right\|, \\
& \left|F_{2}\left(t, x_{1}\right)-F_{2}\left(t, x_{2}\right)\right| \leq k_{2}(t)\left\|x_{1}-x_{2}\right\|,
\end{aligned}
$$

for all $x_{1}, x_{2} \in \mathbb{R}^{n}$ and all $t \in[0, T]$.

Theorem 2.1. Assume that $F=F_{1}+F_{2}$, where $F_{1}, F_{2}$ satisfy assumption $\left(A^{\prime}\right)$ and the following conditions:

(i) $F_{1}(t, \cdot)$ is $(\lambda, \mu)$-subconvex with $\lambda>1 / 2$ and $\mu<2 \lambda^{2}$ for a.e. $t \in[0, T]$;

(ii) there exist $c_{1}, c_{2}>0$ and $\alpha \in[0,1)$ such that

$$
\zeta \in \partial F_{2}(t, x) \Longrightarrow\|\zeta\| \leq c_{1}\|x\|^{\alpha}+c_{2},
$$

for all $x \in \mathbb{R}^{n}$ and a.e. $t \in[0, T]$;

(iii)

$$
\frac{1}{\|x\|^{2 \alpha}}\left[\frac{1}{\mu} \int_{0}^{T} F_{1}(t, \lambda x) d t+\int_{0}^{T} F_{2}(t, x) d t\right] \longrightarrow \infty, \quad \text { as }\|x\| \longrightarrow \infty .
$$

Then problem (2.1) has at least one solution which minimizes $\varphi$ on $H_{T}^{1}$.

Remark 2.2. Theorem 2.1 generalizes [3, Theorem 1]. In fact, [3, Theorem 1] follows from Theorem 2.1 letting $F_{1}=0$.

THEOREM 2.3. Assume that $F=F_{1}+F_{2}$, where $F_{1}, F_{2}$ satisfy assumption $\left(A^{\prime}\right)$ and the following conditions:

(iv) $F_{1}(t, \cdot)$ is $(\lambda, \mu)$-subconvex for a.e. $t \in[0, T]$, and there exists $\gamma \in$ $L^{1}(0, T ; \mathbb{R}), h \in L^{1}\left(0, T ; \mathbb{R}^{n}\right)$ with $\int_{0}^{T} h(t) d t=0$ such that

$$
F_{1}(t, x) \geq\langle h(t), x\rangle+\gamma(t),
$$

for all $x \in \mathbb{R}^{n}$ and a.e. $t \in[0, T]$;

(v) there exist $c_{1}>0, c_{0} \in \mathbb{R}$ such that

$$
\zeta \in \partial F_{2}(t, x) \Longrightarrow\|\zeta\| \leq c_{1},
$$


for all $x \in \mathbb{R}^{n}$ and all $t \in[0, T]$, and

$$
\int_{0}^{T} F_{2}(t, x) d t \geq c_{0}
$$

for all $x \in \mathbb{R}^{n}$

(vi)

$$
\frac{1}{\mu} \int_{0}^{T} F_{1}(t, \lambda x) d t+\int_{0}^{T} F_{2}(t, x) d t \longrightarrow \infty, \quad \text { as }\|x\| \longrightarrow \infty .
$$

Then problem (2.1) has at least one solution which minimizes $\varphi$ on $H_{T}^{1}$.

THEOREM 2.4. Assume that $F=F_{1}+F_{2}$, where $F_{1}, F_{2}$ satisfy assumption $\left(A^{\prime}\right)$ and the following conditions:

(vii) $F_{1}(t, \cdot)$ is $(\lambda, \mu)$-subconvex for a.e. $t \in[0, T]$, and there exists $\gamma \in$ $L^{1}(0, T ; \mathbb{R}), h \in L^{1}\left(0, T ; \mathbb{R}^{n}\right)$ with $\int_{0}^{T} h(t) d t=0$ such that

$$
F_{1}(t, x) \geq\langle h(t), x\rangle+\gamma(t),
$$

for all $x \in \mathbb{R}^{n}$ and a.e. $t \in[0, T]$;

(viii) there exist $c_{1}, c_{2}>0$ and $\alpha \in[0,1)$ such that

$$
\zeta \in \partial F_{2}(t, x) \Longrightarrow\|\zeta\| \leq c_{1}\|x\|^{\alpha}+c_{2},
$$

for all $x \in \mathbb{R}^{n}$ and a.e. $t \in[0, T]$;

(ix)

$$
\frac{1}{\|x\|^{2 \alpha}} \int_{0}^{T} F_{2}(t, x) d t \longrightarrow \infty, \quad \text { as }\|x\| \longrightarrow \infty .
$$

Then problem (2.1) has at least one solution which minimizes $\varphi$ on $H_{T}^{1}$.

\section{Preliminary results}

We introduce some functional spaces. Let $[0, T]$ be a fixed real interval $(0<$ $T<\infty)$ and $1<p<\infty$. We denote by $W_{T}^{1, p}$ the Sobolev space of functions $u \in L^{p}\left(0, T ; \mathbb{R}^{n}\right)$ having a weak derivative $\dot{u} \in L^{p}\left(0, T ; \mathbb{R}^{n}\right)$. The norm over $W_{T}^{1, p}$ is defined by

$$
\|u\|_{W_{T}^{1, p}}=\left(\int_{0}^{T}\|u(t)\|^{p} d t+\int_{0}^{T}\|\dot{u}(t)\|^{p} d t\right)^{1 / p} .
$$

We denote by $H_{T}^{1}$ the Hilbert space $W_{T}^{1,2}$. We recall that

$$
\|u\|_{L^{p}}=\left(\int_{0}^{T}\|u(t)\|^{p} d t\right)^{1 / p}, \quad\|u\|_{\infty}=\max _{t \in[0, T]}\|u(t)\| .
$$

For our aims, it is necessary to recall some very well-known results (for proof and details see [2]): 
Proposition 3.1. If $u \in W_{T}^{1, p}$ then

$$
\|u\|_{\infty} \leq c\|u\|_{W_{T}^{1, p}} .
$$

If $u \in W_{T}^{1, p}$ and $\int_{0}^{T} u(t) d t=0$ then

$$
\|u\|_{\infty} \leq c\|\dot{u}\|_{L^{p}} .
$$

If $u \in H_{T}^{1}$ and $\int_{0}^{T} u(t) d t=0$ then

$$
\begin{aligned}
& \|u\|_{L^{2}} \leq \frac{T}{2 \pi}\|\dot{u}\|_{L^{2}} \quad \text { (Wirtinger's inequality) }, \\
& \|u\|_{\infty}^{2} \leq \frac{T}{12}\|\dot{u}\|_{L^{2}}^{2} \quad \text { (Sobolev inequality). }
\end{aligned}
$$

Proposition 3.2. If the sequence $\left(u_{k}\right)_{k}$ converges weakly to $u$ in $W_{T}^{1, p}$, then $\left(u_{k}\right)_{k}$ converges uniformly to $u$ on $[0, T]$.

Let $X$ be a Banach space. Now, following [1], for each $x, v \in X$, we define the generalized directional derivative at $x$ in the direction $v$ of a given $f \in$ $\operatorname{Lip}_{\text {loc }}(X, \mathbb{R})$ as

$$
f^{0}(x ; v)=\limsup _{y \rightarrow x, \lambda \searrow 0} \frac{f(y+\lambda v)-f(y)}{\lambda}
$$

and denote $x$ by

$$
\partial f(x)=\left\{x^{*} \in X^{*}: f^{0}(x ; v) \geq\left\langle x^{*}, v\right\rangle, \forall v \in X\right\}
$$

the generalized gradient of $f$ at $x$ (the Clarke subdifferential).

We recall the Lebourg's mean value theorem (see [1, Theorem 2.3.7]). Let $x$ and $y$ be points in $X$, and suppose that $f$ is Lipschitz on an open set containing the line segment $[x, y]$. Then there exists a point $u$ in $(x, y)$ such that

$$
f(y)-f(x) \in\langle\partial f(u), y-x\rangle .
$$

Clarke considered in [1] the following abstract framework:

- let $(T, \mathscr{T}, \mu)$ be a positive complete measure space with $\mu(T)<\infty$, and let $Y$ be a separable Banach space;

- let $Z$ be a closed subspace of $L^{p}(T ; Y)$ (for some $p$ in $[1, \infty)$ ), where $L^{p}(T ; Y)$ is the space of $p$-integrable functions from $T$ to $Y$;

- we define a functional $f$ on $Z$ via

$$
f(x)=\int_{T} f_{t}(x(t)) \mu(d t),
$$

where $f_{t}: Y \rightarrow R,(t \in T)$ is a given family of functions; 
- we suppose that for each $y$ in $Y$ the function $t \rightarrow f_{t}(y)$ is measurable, and that $x$ is a point at which $f(x)$ is defined (finitely).

Hypothesis 3.3. There is a function $k$ in $L^{q}(T, R),(1 / p+1 / q=1)$ such that, for all $t \in T$,

$$
\left|f_{t}\left(y_{1}\right)-f_{t}\left(y_{2}\right)\right| \leq k(t)\left\|y_{1}-y_{2}\right\|_{Y} \quad \forall y_{1}, y_{2} \in Y \text {. }
$$

Hypothesis 3.4. Each function $f_{t}$ is Lipschitz (of some rank) near each point of $Y$, and for some constant $c$, for all $t \in T, y \in Y$, one has

$$
\zeta \in \partial f_{t}(y) \Longrightarrow\|\zeta\|_{Y^{*}} \leq c\left\{1+\|y\|_{Y}^{p-1}\right\} \text {. }
$$

Under the conditions described above Clarke proved (see [1, Theorem 2.7.5]):

THEOREM 3.5. Under either of Hypotheses 3.3 or 3.4, $f$ is uniformly Lipschitz on bounded subsets of $Z$, and there is

$$
\partial f(x) \subset \int_{T} \partial f_{t}(x(t)) \mu(d t) .
$$

Further, if each $f_{t}$ is regular at $x(t)$ then $f$ is regular at $x$ and equality holds.

Remark 3.6. The function $f$ is globally Lipschitz on $Z$ when Hypothesis 3.3 holds.

Now we can prove the following result.

Theorem 3.7. Let $F:[0, T] \times \mathbb{R}^{n} \rightarrow \mathbb{R}$ such that $F=F_{1}+F_{2}$ where $F_{1}$, $F_{2}$ are measurable in $t$ for each $x \in \mathbb{R}^{n}$, and there exist $k_{1} \in L^{2}(0, T ; \mathbb{R})$, $a \in C\left(\mathbb{R}^{+}, \mathbb{R}^{+}\right), b \in L^{1}\left(0, T ; \mathbb{R}^{+}\right), c_{1}, c_{2}>0$, and $\alpha \in[0.1)$ such that

$$
\begin{gathered}
\left|F_{1}\left(t, x_{1}\right)-F_{1}\left(t, x_{2}\right)\right| \leq k_{1}(t)\left\|x_{1}-x_{2}\right\|, \\
\left|F_{2}(t, x)\right| \leq a(\|x\|) b(t), \\
\zeta \in \partial F_{2}(t, x) \Longrightarrow\|\zeta\| \leq c_{1}\|x\|^{\alpha}+c_{2},
\end{gathered}
$$

for all $t \in[0, T]$ and all $x, x_{1}, x_{2} \in \mathbb{R}^{n}$. We suppose that $L:[0, T] \times \mathbb{R}^{n} \times \mathbb{R}^{n} \rightarrow$ $\mathbb{R} i$ given by $L(t, x, y)=(1 / 2)\|y\|^{2}+F(t, x)$.

Then, the functional $f: Z \in \mathbb{R}$, where

$$
Z=\left\{(u, v) \in L^{2}(0, T ; Y): u(t)=\int_{0}^{t} v(s) d s+c, c \in \mathbb{R}^{n}\right\}
$$

given by $f(u, v)=\int_{0}^{T} L(t, u(t), v(t)) d t$, is uniformly Lipschitz on bounded subsets of $Z$ and

$$
\partial f(u, v) \subset \int_{0}^{T}\left\{\partial F_{1}(t, u(t))+\partial F_{2}(t, u(t))\right\} \times\{v(t)\} d t .
$$


Proof. Let $L_{1}(t, x, y)=F_{1}(t, x), L_{2}(t, x, y)=(1 / 2)\|y\|^{2}+F_{2}(t, x)$, and $f_{1}, f_{2}$ : $Z \rightarrow \mathbb{R}$ given by $f_{1}(u, v)=\int_{0}^{T} L_{1}(t, u(t), v(t)) d t, f_{2}(u, v)=\int_{0}^{T} L_{2}(t, u(t)$, $v(t)) d t$. For $f_{1}$ we can apply Theorem 3.5 under Hypothesis 3.3, with the following cast of characters:

- $(T, \mathscr{T}, \mu)=[0, T]$ with Lebesgue measure, $Y=\mathbb{R}^{n} \times \mathbb{R}^{n}$ is the Hilbert product space (hence is separable);

- $p=2$ and

$$
Z=\left\{(u, v) \in L^{2}(0, T ; Y): u(t)=\int_{0}^{t} v(s) d s+c, c \in \mathbb{R}^{n}\right\}
$$

is a closed subspace of $L^{2}(0, T ; Y)$;

- $f_{t}(x, y)=L_{1}(t, x, y)=F_{1}(t, x)$; in our assumptions it results that the integrand $L_{1}(t, x, y)$ is measurable in $t$ for a given element $(x, y)$ of $Y$ and there exists $k \in L^{2}(0, T ; \mathbb{R})$ such that

$$
\begin{aligned}
\left|L_{1}\left(t, x_{1}, y_{1}\right)-L_{1}\left(t, x_{2}, y_{2}\right)\right| & =\left|F_{1}\left(t, x_{1}\right)-F_{1}\left(t, x_{2}\right)\right| \\
& \leq k_{1}(t)\left\|x_{1}-x_{2}\right\| \\
& \leq k_{1}(t)\left(\left\|x_{1}-x_{2}\right\|+\left\|y_{1}-y_{2}\right\|\right) \\
& =k_{1}(t)\left\|\left(x_{1}, y_{1}\right)-\left(x_{2}, y_{2}\right)\right\|_{Y},
\end{aligned}
$$

for all $t \in[0, T]$ and all $\left(x_{1}, y_{1}\right),\left(x_{2}, y_{2}\right) \in Y$. Hence $f_{1}$ is uniformly Lipschitz on bounded subsets of $Z$ and one has

$$
\partial f_{1}(u, v) \subset \int_{0}^{T} \partial L_{1}(t, u(t), v(t)) d t .
$$

For $f_{2}$ we can apply Theorem 3.5 under Hypothesis 3.4 with the same cast of characters, but now $f_{t}(x, y)=L_{2}(t, x, y)=(1 / 2)\|y\|^{2}+F_{2}(t, x)$. In our assumptions, it results that the integrand $L_{2}(t, x, y)$ is measurable in $t$ for a given element $(x, y)$ of $Y$ and locally Lipschitz in $(x, y)$ for each $t \in[0, T]$.

Proposition 2.3.15 in [1] implies

$$
\partial L_{2}(t, x, y) \subset \partial_{x} L_{2}(t, x, y) \times \partial_{y} L_{2}(t, x, y)=\partial F_{2}(t, x) \times y .
$$

Using (3.15) and (3.21), if $\zeta=\left(\zeta_{1}, \zeta_{2}\right) \in \partial L_{2}(t, x, y)$ then $\zeta_{1} \in \partial F_{2}(t, x)$ and $\zeta_{2}=y$, and hence

$$
\|\zeta\|=\left\|\zeta_{1}\right\|+\left\|\zeta_{2}\right\| \leq c_{1}\|x\|^{\alpha}+c_{2}+\|y\| \leq \tilde{c}\{1+\|(x, y)\|\},
$$

for each $t \in[0, T]$. Hence $f_{2}$ is uniformly Lipschitz on bounded subsets of $Z$ and one has

$$
\partial f_{2}(u, v) \subset \int_{0}^{T} \partial L_{2}(t, u(t), v(t)) d t
$$


It follows that $f=f_{1}+f_{2}$ is uniformly Lipschitz on the bounded subsets of $Z$.

Propositions 2.3.3 and 2.3.15 in [1] imply that

$$
\begin{aligned}
\partial f(u, v) \subset \partial f_{1}(u, v)+\partial f_{2}(u, v) \\
\subset \int_{0}^{T}\left[\partial L_{1}(t, u(t), v(t))+\partial L_{2}(t, u(t), v(t))\right] d t \\
\subset \int_{0}^{T}\left[\left(\partial_{x} L_{1}(t, u(t), v(t)) \times \partial_{y} L_{1}(t, u(t), v(t))\right)\right. \\
\left.\quad+\left(\partial_{x} L_{2}(t, u(t), v(t)) \times \partial_{y} L_{2}(t, u(t), v(t))\right)\right] d t \\
\subset \int_{0}^{T}\left[\left(\partial_{x} L_{1}(t, u(t), v(t))+\partial_{x} L_{2}(t, u(t), v(t))\right)\right. \\
\left.\quad \times\left(\partial_{y} L_{1}(t, u(t), v(t))+\partial_{y} L_{2}(t, u(t), v(t))\right)\right] d t \\
=\int_{0}^{T}\left(\partial F_{1}(t, u(t))+\partial F_{2}(t, u(t))\right) \times\{v(t)\} d t .
\end{aligned}
$$

Moreover, Corollary 1 of Proposition 2.3.3 in [1] implies that, if at least one of the functions $F_{1}, F_{2}$ is strictly differentiable in $x$ for all $t \in[0, T]$ then

$$
\partial f(u, v) \subset \int_{0}^{T} \partial F(t, u(t)) \times\{v(t)\} d t .
$$

Remark 3.8. The interpretation of expression (3.25) is that if $\left(u_{0}, v_{0}\right)$ is an element of $Z$ (so that $\left.v_{0}=\dot{u}_{0}\right)$ and if $\zeta \in \partial f\left(u_{0}, v_{0}\right)$, we deduce the existence of a measurable function $(q(t), p(t))$ such that

$$
q(t) \in \partial F\left(t, u_{0}(t)\right), \quad p(t)=v_{0}(t) \quad \text { a.e. on }[0, T]
$$

and for any $(u, v)$ in $Z$, one has

$$
\langle\zeta,(u, v)\rangle=\int_{0}^{T}\{\langle q(t), u(t)\rangle+\langle p(t), v(t)\rangle\} d t .
$$

In particular, if $\zeta=0$ (so that $u_{0}$ is a critical point for $\varphi(u)=\int_{0}^{T}\left[(1 / 2)\|\dot{u}(t)\|^{2}+\right.$ $F(t, u(t))] d t)$, it then follows easily that $q(t)=\dot{p}(t)$ a.e., or taking into account (3.26)

$$
\ddot{u}_{0}(t) \in \partial F\left(t, u_{0}(t)\right) \quad \text { a.e. on }[0, T],
$$

so that $u_{0}$ satisfies the inclusions system (2.1).

Remark 3.9. Of course, if $F$ is continuously differentiable in $x$, then system (2.1) becomes system (1.1). 


\section{Proofs of the theorems}

Proof of Theorem 2.1. From assumption $\left(\mathrm{A}^{\prime}\right)$ it follows immediately that there exist $a \in C\left(\mathbb{R}^{+}, \mathbb{R}^{+}\right), b \in L^{1}\left(0, T ; \mathbb{R}^{+}\right)$such that

$$
\left|F_{1}(t, x)\right| \leq a(\|x\|) b(t)
$$

for all $x \in \mathbb{R}^{n}$ and all $t \in[0, T]$. Like, in [4], we obtain

$$
F_{1}(t, x) \leq\left(2 \mu\|x\|^{\beta}+1\right) a_{0} b(t),
$$

for all $x \in \mathbb{R}^{n}$ and all $t \in[0, T]$, where $\beta<2$ and $a_{0}=\max _{0 \leq s \leq 1} a(s)$.

For $u \in H_{T}^{1}$, let $\bar{u}=(1 / T) \int_{0}^{T} u(t) d t$ and $\tilde{u}=u-\bar{u}$. From Lebourg's mean value theorem it follows that for each $t \in[0, T]$ there exist $z(t)$ in $(\bar{u}, u(t))$ and $\zeta \in \partial F_{2}(t, z(t))$ such that $F_{2}(t, u(t))-F_{2}(t, \bar{u})=\langle\zeta, \tilde{u}(t)\rangle$. It follows from (2.3) and Sobolev's inequality that

$$
\begin{aligned}
\mid \int_{0}^{T} & {\left[F_{2}(t, u(t))-F_{2}(t, \bar{u})\right] d t \mid } \\
& \leq \int_{0}^{T}\left|F_{2}(t, u(t))-F_{2}(t, \bar{u})\right| d t \leq \int_{0}^{T}\|\zeta\|\|\tilde{u}(t)\| d t \\
& \leq \int_{0}^{T}\left[2 c_{1}\left(\|\bar{u}\|^{\alpha}+\|\tilde{u}(t)\|^{\alpha}\right)+c_{2}\right]\|\tilde{u}(t)\| d t \\
& \leq 2 c_{1} T\|\tilde{u}\|_{\infty}\|\bar{u}\|^{\alpha}+2 c_{1} T\|\tilde{u}\|_{\infty}^{\alpha+1}+c_{2} T\|\tilde{u}\|_{\infty} \\
& \leq \frac{3}{T}\|\tilde{u}\|_{\infty}^{2}+\frac{T^{3}}{3} c_{1}^{2}\|\bar{u}\|^{2 \alpha}+2 c_{1} T\|\tilde{u}\|_{\infty}^{\alpha+1}+c_{2} T\|\tilde{u}\|_{\infty} \\
& \leq \frac{1}{4}\|\dot{u}\|_{L^{2}}^{2}+C_{1}\|\dot{u}\|_{L^{2}}^{\alpha+1}+C_{2}\|\dot{u}\|_{L^{2}}+C_{3}\|\bar{u}\|^{2 \alpha},
\end{aligned}
$$

for all $u \in H_{T}^{1}$ and some positive constants $C_{1}, C_{2}$, and $C_{3}$. Hence we have

$$
\begin{aligned}
\varphi(u) \geq & \frac{1}{2} \int_{0}^{T}\|\dot{u}(t)\|^{2} d t+\frac{1}{\mu} \int_{0}^{T} F_{1}(t, \lambda \bar{u}) d t-\int_{0}^{T} F_{1}(t,-\tilde{u}(t)) d t \\
& +\int_{0}^{T} F_{2}(t, \bar{u}) d t+\int_{0}^{T}\left[F_{2}(t, u(t))-F_{2}(t, \bar{u})\right] d t \\
\geq & \frac{1}{4}\|\dot{u}\|_{L^{2}}^{2}-C_{1}\|\dot{u}\|_{L^{2}}^{\alpha+1}-C_{2}\|\dot{u}\|_{L^{2}}-C_{3}\|\bar{u}\|^{2 \alpha}-\left(2 \mu\|\tilde{u}\|_{\infty}^{\beta}+1\right) \int_{0}^{T} a_{0} b(t) d t \\
& +\frac{1}{\mu} \int_{0}^{T} F_{1}(t, \lambda \bar{u}) d t+\int_{0}^{T} F_{2}(t, \bar{u}) d t
\end{aligned}
$$




$$
\begin{aligned}
\geq & \frac{1}{4}\|\dot{u}\|_{L^{2}}^{2}-C_{1}\|\dot{u}\|_{L^{2}}^{\alpha+1}-C_{2}\|\dot{u}\|_{L^{2}}-C_{4}\|\dot{u}\|_{L^{2}}^{\beta}-C_{5} \\
& +\|\bar{u}\|^{2 \alpha}\left\{\frac{1}{\|\bar{u}\|^{2 \alpha}}\left[\frac{1}{\mu} \int_{0}^{T} F_{1}(t, \lambda \bar{u}) d t+\int_{0}^{T} F_{2}(t, \bar{u}) d t\right]-C_{3}\right\}
\end{aligned}
$$

for all $u \in H_{T}^{1}$, which implies that $\varphi(u) \rightarrow \infty$ as $\|u\| \rightarrow \infty$ by (2.4) because $\alpha<1, \beta<2$, and the norm $\|u\|=\left(\|\bar{u}\|^{2}+\|\dot{u}\|_{L^{2}}^{2}\right)^{1 / 2}$ is an equivalent norm on $H_{T}^{1}$. Now we write $\varphi(u)=\varphi_{1}(u)+\varphi_{2}(u)$ where

$$
\varphi_{1}(u)=\frac{1}{2} \int_{0}^{T}\|\dot{u}(t)\|^{2} d t, \quad \varphi_{2}(u)=\int_{0}^{T} F(t, u(t)) d t .
$$

The function $\varphi_{1}$ is weakly lower semi-continuous (w.l.s.c.) on $H_{T}^{1}$. From (i), (ii), and Theorem 3.5, taking into account Remark 3.6 and Proposition 3.2, it follows that $\varphi_{2}$ is w.l.s.c. on $H_{T}^{1}$. By [2, Theorem 1.1], it follows that $\varphi$ has a minimum $u_{0}$ on $H_{T}^{1}$. Evidently, $Z \simeq H_{T}^{1}$ and $\varphi(u)=f(u, v)$ for all $(u, v) \in Z$. From Theorem 3.7, it results that $f$ is uniformly Lipschitz on bounded subsets of $Z$, and therefore $\varphi$ possesses the same properties relative to $H_{T}^{1}$. Proposition 2.3.2 in [1] implies that $0 \in \partial \varphi\left(u_{0}\right)$ (so that $u_{0}$ is a critical point for $\varphi$ ). Now from Theorem 3.7 and Remark 3.8 it follows that problem (2.1) has at least one solution $u \in H_{T}^{1}$.

Proof of Theorem 2.3. Let $\left(u_{k}\right)$ be a minimizing sequence of $\varphi$. It follows from (iv), (v), Lebourg's mean value theorem, and Sobolev inequality, that

$$
\begin{aligned}
\varphi\left(u_{k}\right) \geq & \frac{1}{2}\left\|\dot{u}_{k}\right\|_{L^{2}}^{2}+\int_{0}^{T}\left\langle h(t), u_{k}(t)\right\rangle d t+\int_{0}^{T} \gamma(t) d t \\
& +\int_{0}^{T} F_{2}\left(t, \bar{u}_{k}\right) d t-\int_{0}^{T}\|\zeta\|\left\|\tilde{u}_{k}(t)\right\| d t \\
\geq & \frac{1}{2}\left\|\dot{u}_{k}\right\|_{L^{2}}^{2}-\left\|\tilde{u}_{k}\right\|_{\infty} \int_{0}^{T}\|h(t)\| d t \\
& +\int_{0}^{T} \gamma(t) d t-c_{1}\left\|\tilde{u}_{k}\right\|_{\infty}+c_{0} \\
\geq & \frac{1}{2}\left\|\dot{u}_{k}\right\|_{L^{2}}^{2}-c_{2}\left\|\dot{u}_{k}\right\|_{L^{2}}-c_{3},
\end{aligned}
$$

for all $k$ and some constants $c_{2}, c_{3}$, which implies that $\left(\tilde{u}_{k}\right)$ is bounded. On the other hand, in a way similar to the proof of Theorem 2.1, one has

$$
\left|\int_{0}^{T}\left[F_{2}(t, u(t))-F_{2}(t, \bar{u})\right] d t\right| \leq \frac{1}{4}\|\dot{u}\|_{L^{2}}^{2}+C_{1}\|\dot{u}\|_{L^{2}},
$$


for all $k$ and some positive constant $C_{1}$, which implies that

$$
\begin{aligned}
\varphi\left(u_{k}\right) \geq & \frac{1}{2}\left\|\dot{u}_{k}\right\|_{L^{2}}^{2}+\frac{1}{\mu} \int_{0}^{T} F_{1}\left(t, \lambda \bar{u}_{k}\right) d t-\int_{0}^{T} F_{1}\left(t,-\tilde{u}_{k}(t)\right) d t \\
& +\int_{0}^{T} F_{2}\left(t, \bar{u}_{k}\right) d t+\int_{0}^{T}\left[F_{2}(t, u(t))-F_{2}\left(t, \bar{u}_{k}\right)\right] d t \\
\geq & \frac{1}{4}\left\|\dot{u}_{k}\right\|_{L^{2}}^{2}-a\left(\left\|\tilde{u}_{k}\right\|_{\infty}\right) \int_{0}^{T} b(t) d t-C_{1}\left\|\dot{u}_{k}\right\|_{L^{2}} \\
& +\frac{1}{\mu} \int_{0}^{T} F_{1}\left(t, \lambda \bar{u}_{k}\right) d t+\int_{0}^{T} F_{2}\left(t, \bar{u}_{k}\right) d t,
\end{aligned}
$$

for all $k$ and some positive constant $C_{1}$. It follows from (vi) and the boundedness of $\left(\tilde{u}_{k}\right)$ that $\left(\bar{u}_{k}\right)$ is bounded. Hence $\varphi$ has a bounded minimizing sequence $\left(u_{k}\right)$. This completes the proof.

Proof of Theorem 2.4. From (vii), (3.26), and Sobolev's inequality it follows that

$$
\begin{aligned}
\varphi(u) \geq & \frac{1}{2}\|\dot{u}\|_{L^{2}}^{2}+\int_{0}^{T}\langle h(t), u(t)\rangle d t+\int_{0}^{T} \gamma(t) d t \\
& +\int_{0}^{T} F_{2}(t, \bar{u}) d t+\int_{0}^{T}\left[F_{2}(t, u(t))-F_{2}(t, \bar{u})\right] d t \\
\geq & \frac{1}{4}\|\dot{u}\|_{L^{2}}^{2}-\|\tilde{u}\|_{\infty} \int_{0}^{T}\|h(t)\| d t+\int_{0}^{T} \gamma(t) d t \\
& -C_{1}\|\dot{u}\|_{L^{2}}^{\alpha+1}-C_{2}\|\dot{u}\|_{L^{2}}+\int_{0}^{T} F_{2}(t, \bar{u}) d t-C_{3}\|\bar{u}\|^{2 \alpha} \\
\geq & \frac{1}{4}\|\dot{u}\|_{L^{2}}^{2}-C_{1}\|\dot{u}\|_{L^{2}}^{\alpha+1}-C_{4}\left(\|\dot{u}\|_{L^{2}}+1\right) \\
& +\|\bar{u}\|^{2 \alpha}\left[\frac{1}{\|\bar{u}\|^{2 \alpha}} \int_{0}^{T} F_{2}(t, \bar{u}) d t-C_{3}\right]
\end{aligned}
$$

for all $u \in H_{T}^{1}$ and some positive constants $C_{1}, C_{3}$, and $C_{4}$. Now it follows like in the proof of Theorem 2.1 that $\varphi$ is coercive by (ix), which completes the proof.

\section{References}

[1] F. H. Clarke, Optimization and Nonsmooth Analysis, 2nd ed., Classics in Applied Mathematics, vol. 5, SIAM, Pennsylvania, 1990. MR 91e:49001. Zbl 696.49002.

[2] J. Mawhin and M. Willem, Critical Point Theory and Hamiltonian Systems, Applied Mathematical Sciences, vol. 74, Springer-Verlag, New York, 1989. MR 90e:58016. Zbl 676.58017. 
[3] D. Pasca, Periodic solutions for second order differential inclusions with sublinear nonlinearity, Panamer. Math. J. 10 (2000), no. 4, 35-45. CMP 1801529.

[4] X.-P. Wu and C.-L. Tang, Periodic solutions of a class of non-autonomous second-order systems, J. Math. Anal. Appl. 236 (1999), no. 2, 227-235. MR 2000g:34069. Zbl 991.21227.

Daniel Paşca: Department of Mathematics, University of Oradea, Armatei Romane 5, 3700, Oradea, Romania

E-mail address: pasca@mathematik.uni-kl.de 


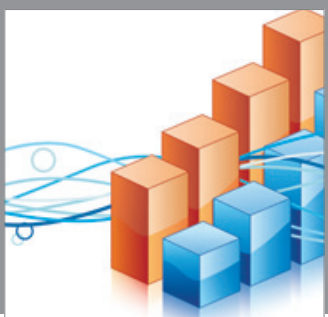

Advances in

Operations Research

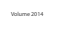

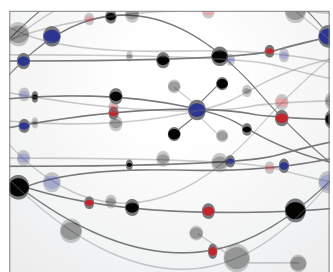

\section{The Scientific} World Journal
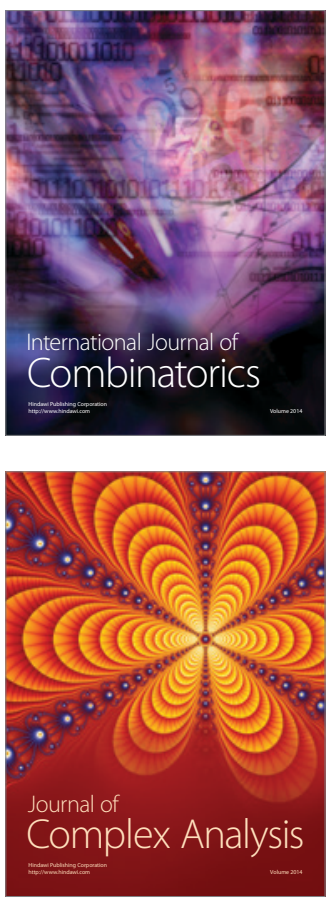

International Journal of

Mathematics and

Mathematical

Sciences
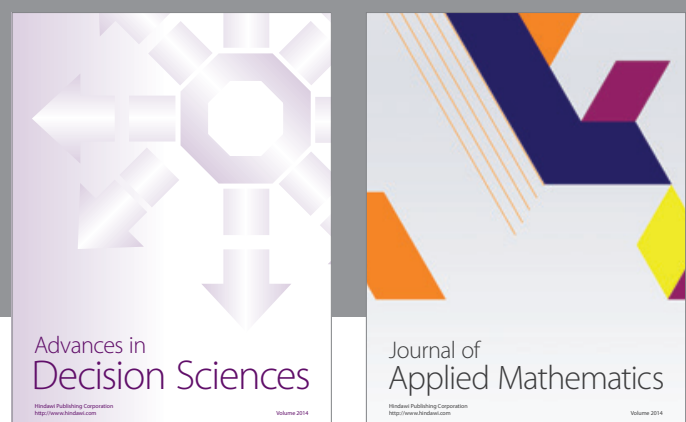

Journal of

Applied Mathematics
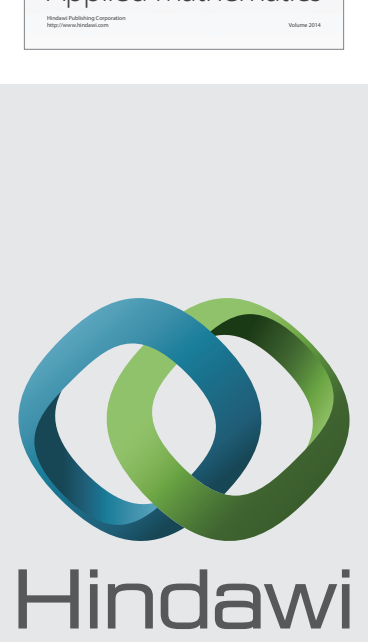

Submit your manuscripts at http://www.hindawi.com
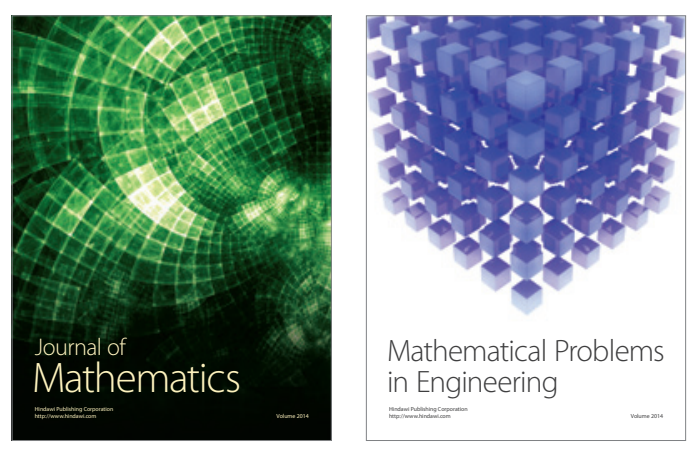

Mathematical Problems in Engineering
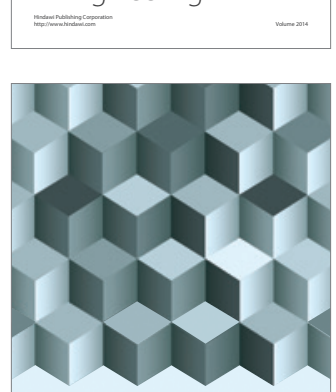

Journal of

Function Spaces
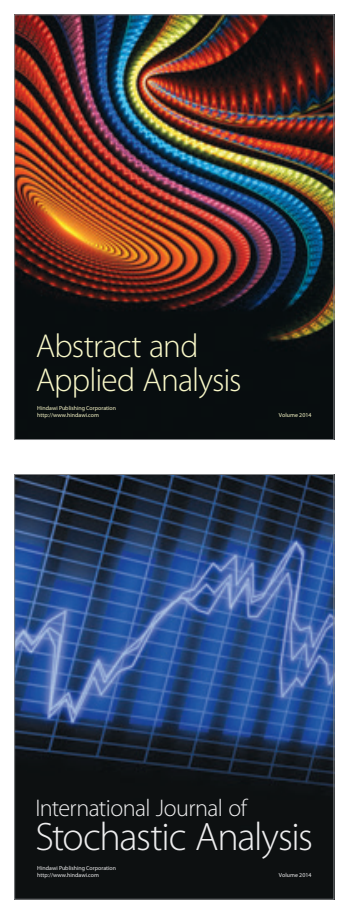

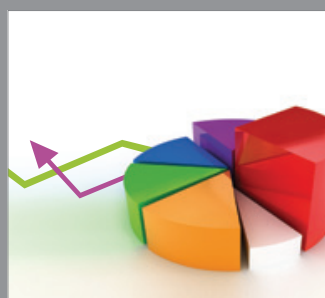

ournal of

Probability and Statistics

Promensencen
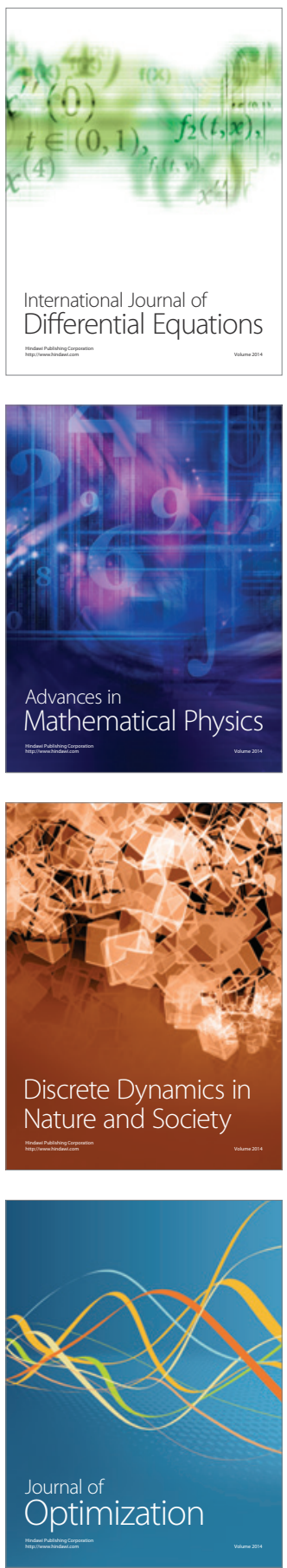